\title{
Thermodynamic Aspects of Homogeneous Nucleation Enhanced by Icosahedral Short Range Order in Liquid Fcc-Type Alloys
}

\author{
MICHEL RAPPAZ ${ }^{1,2}$ and GÜVEN KURTULDU ${ }^{1}$ \\ 1.-Computational Materials Laboratory, Institute of Materials, Ecole Polytechnique Fédérale de \\ Lausanne, Station 12, 1015 Lausanne, Switzerland. 2.—e-mail: michel.rappaz@epfl.ch
}

We have recently shown that minute solute element additions to liquid metallic alloys can strongly influence the nucleation of the fcc phase and act as a grain refinement method. Electron back-scattered diffraction observations revealed a concomitant increase in the percentage of nearest neighbor $(\mathrm{nn})$ grains that are in a twin relationship. Furthermore, multiple-twinned (MT) nn grain configurations with a fivefold symmetry around a common $\langle 110\rangle$ direction have been identified, an occurrence that can be explained when the symmetry of the icosahedron is accounted for. It was then conjectured that a new nucleation mechanism occurs in two steps: first, the formation of small icosahedral quasicrystals in the melt, followed by heteroepitaxy of the fcc phase on facets of these quasicrystals. In the present contribution, based on thermodynamics arguments, it is proposed that the first step occurs by spinodal decomposition of the liquid, in a manner similar to Guinier-Preston zones formation in solid state precipitation, while the second step is a transformation of these quasicrystal precursors into MT-fcc nanocrystals once the driving force for this transformation is sufficient to overcome the fcc-liquid interfacial energy and the elastic strains associated with MT-fcc nanoparticles. This explanation sets up guidelines for finding solute elements and composition ranges that favor this grain refinement mechanism.

\section{INTRODUCTION}

In two recent papers, ${ }^{1,2}$ we reported the discovery of a new nucleation mechanism for the solidification of fcc-type metallic alloys. First observed in Al-20 wt.\% Zn melts, ${ }^{1}$ it was shown that the addition of $0.1 \mathrm{wt} . \%$ chromium drastically reduces the grain size in samples that were solidified under nearly uniform temperature conditions (equiaxed solidification). Furthermore, a substantial fraction of grain boundaries (about $2 \%$ ) were identified by electron back-scattered diffraction (EBSD) as incoherent twin (or near-twin) boundaries. This percentage is much larger than that expected for randomly oriented grains (with the prescribed identification accuracy of $5^{\circ}$ ). Some of them were near-twin boundary, i.e., a twin orientation relationship but with a rotation of about $5-7^{\circ}$ around a common $\langle 110\rangle$ direction. Since twin and near-twin orientation relationships naturally appear when 5 fcc tetrahedra are assembled into a decahedron (or icosahedron), for which there is a missing gap of about $7.5^{\circ}$, configurations of multiple fcc nearestneighbor (nn) grains sharing the fivefold symmetry of the icosahedron were sought and found.

Based on these observations, a new (homogeneous) nucleation mechanism was then conjectured for Al-Zn:Cr alloys (Fig. 1). Already suggested by Frank, ${ }^{3}$ atoms in metallic liquids can develop icosahedral short-range order (ISRO), as shown in Fig. 1a. This atomic configuration was confirmed later by molecular dynamics simulations ${ }^{4,5}$ and small-angle scattering in various metallic liquids. ${ }^{6-9}$ This configuration is key to the understanding of quasicrystals (QC) formation, a discovery made first by Shechtman et al. ${ }^{10}$ in Al-Mn alloys, but then in many other alloys including Al-Cr. ${ }^{11-13}$ Icosahedral order is also directly observed in the many building blocks of the monoclinic unit cell of the approximant $\mathrm{Al}_{45} \mathrm{Cr}_{7}$ intermetallic phase. ${ }^{14,15}$ Due to their low 


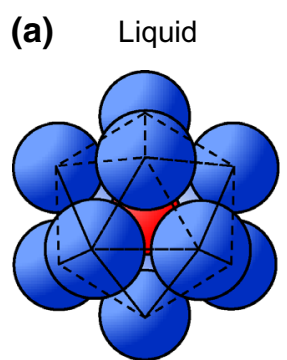

(b) Liquid + iQC

(c) Liquid + iQC $+\alpha$

(d) Liquid $+\alpha$
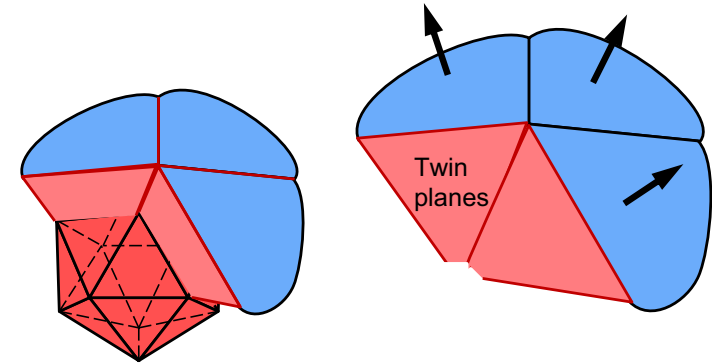

Fig. 1. Heteroepitaxy nucleation mechanism of the fcc phase on iQC for the case of Al-Zn:Cr alloys: (a) Frank's icosahedral short-range order of atoms in the liquid (red atom: $\mathrm{Cr}$, blue atoms: $\mathrm{Al}$ or $\mathrm{Zn}$ ); (b) formation of iQC in the liquid; (c) heteroepitaxy of the $\alpha$-fcc phase on the iQC facets, with twin planes in between the various $\alpha$ nuclei; (d) growth of the fcc phase and dissolution of the iQC during cooling due to the peritectic nature of the phase diagram (Color figure online).

interfacial energy with the liquid, ${ }^{16} \mathrm{QCs}$ can form with a very low nucleation barrier and then grow (Fig. 1b). Once the peritectic temperature is reached, the mechanism proceeds by heteroepitaxy formation of the fcc phase on the facets of the icosahedron, with multiple twin (MT) relationships as shown in Fig. 1c. The heteroepitaxy relationship between the QC template and the fcc phase is: $\langle 111\rangle_{\text {fcc }} \|$ threefold symmetry axis of $\mathrm{QC}$ and $\langle 110\rangle_{\text {fcc }} \perp$ twofold symmetry axis of QC. Upon further cooling, the MT fcc grains grow as indicated in Fig. 1d and the peritectic transformation can even transform ("kill") the QC template (a "perfect crime", since the nucleation template disappears after it has performed its duty!). Once the fcc phase reaches a critical radius, the solid-liquid interface can become unstable, leading to the formation of dendrites. However, two fcc solids issued from two nuclei formed by heteroepitaxy on adjacent facets of a $\mathrm{QC}$ are in a twin (or near-twin) relationship, thus producing a twinned dendrite, i.e., a dendrite made of a twinned and untwinned side separated by a twin plane in their trunk center. ${ }^{1,17,18}$ This morphology has been well known in direct chill cast industrial $\mathrm{Al}$ alloys for more than 60 years, although its origin could not be explained before this nucleation mechanism was discovered.

The same nucleation mechanism was then shown to also be responsible for the extreme grain refinement in yellow gold alloys (Au-12.5 wt.\%Cu12.5 wt. $\% \mathrm{Ag})$, when minute amounts of Ir (5-200 ppm by weight) are added to the melt. ${ }^{2}$ This has been a common industrial practice for jewelry applications for nearly half a century. ${ }^{19-21}$ In this case, a configuration of 9 MT-nn fcc grains reproducing the six fivefold symmetry axes of the icosahedron was found.

This paper further investigates this nucleation mechanism from a thermodynamic point of view, after a brief summary in the following section of the experimental findings of Refs. 1 and 2. First, a thermodynamic analogy is made between the nucleation of QCs in the liquid and the formation of Guinier-Preston zones in solid state transformations. If a specific Gibbs free energy can be set for the approximant intermetallic phase, we suggest that one may view $\mathrm{QC}$ formation as a spinodal decomposition of the liquid, leading to zones both rich and lean in icosahedral building blocks (e.g., rich and lean of $\mathrm{Cr}$ for $\mathrm{Al}-\mathrm{Zn}$ alloy). It is then shown that the QC-heteroepitaxy nucleation mechanism assumed in Refs. 1 and 2 (Fig. 1) does not satisfy a positive Gibbs free energy balance when the interfacial energy and the elastic strain energy are accounted for. Another mechanism is then suggested by which the iQCs transform into MT fcc grains via a peritectic transformation. According to this mechanism, guidelines for finding the best conditions under which QC-induced nucleation in fcc liquid metals is effective are suggested.

\section{SUMMARY OF THE EXPERIMENTAL FINDINGS IN Al-Zn:Cr and Au-Ag-Cu:Ir ALLOYS}

In this section, we briefly summarize in two figures the findings reported in Refs. 1 and 2 for $\mathrm{Al}-\mathrm{Zn}: \mathrm{Cr}$ and Au-Ag-Cu:Ir, respectively. Figure 2 shows the grain refinement effect induced in $\mathrm{Al}-20 \mathrm{wt} . \% \mathrm{Zn}$ (top) and in Au-12.5 wt.\%Ag$12.5 \mathrm{wt} . \% \mathrm{Cu}$ (bottom) alloys by the addition of 0.1 wt. $\% \mathrm{Cr}$ and $0.02 \mathrm{wt} . \% \mathrm{Ir}$, respectively. The microstructures are represented by false color EBSD maps. All the experimental details can be found in Refs. 1 and 2. This grain refinement effect, hitherto unknown in the aluminum industry, is commonly used in the jewelry industry, but without knowledge of the underlying mechanism. In these false color EBSD maps, two nn grains that are in a twin relationship have their boundary displayed in white. In the grain-refined aluminum sample, about $2 \%$ of the grain boundaries correspond to (incoherent) twins, whereas the percentage is about $11 \%$ in the yellow gold specimen. This percentage is much larger than what can be expected from a random distribution of grain orientation (with a tolerance of $5^{\circ}$ ).

More importantly, many configuration of MT-nn grains can be found in both type of alloys when they are grain refined with $\mathrm{Cr}$ or Ir. Figure 3 a shows 5 grains in Al-Zn:Cr sharing a nearly common $\langle 110\rangle$ orientation in a MT fivefold symmetry configuration. The traces of their common twin (or near-twin) $\{111\}$ planes are shown with arcs of circle in 
(a) Al-Zn

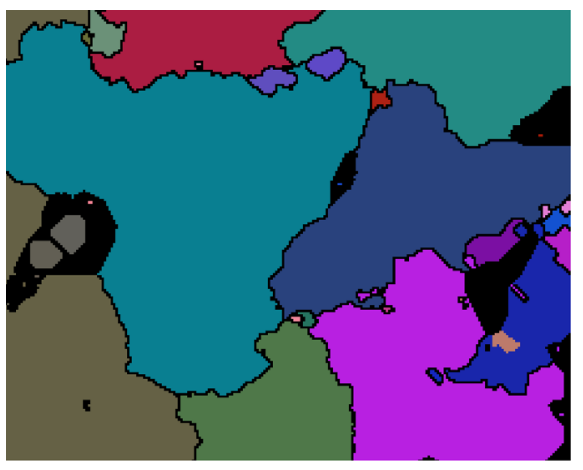

$0.5 \mathrm{~mm}$

(c) Au-Cu-Ag

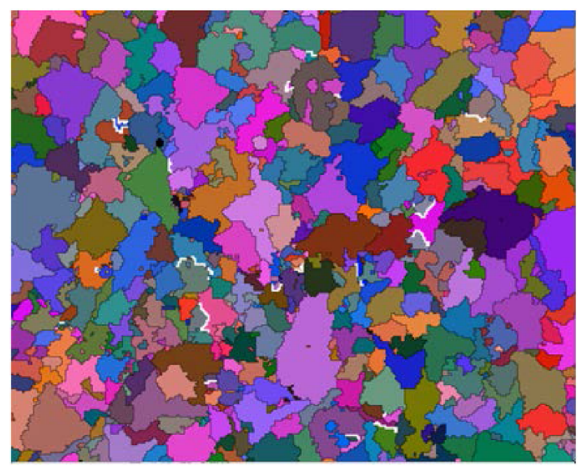

$2 \mathrm{~mm}$ (b) $\mathrm{Al}-\mathrm{Zn}+1000 \mathrm{ppm} \mathrm{Cr}$

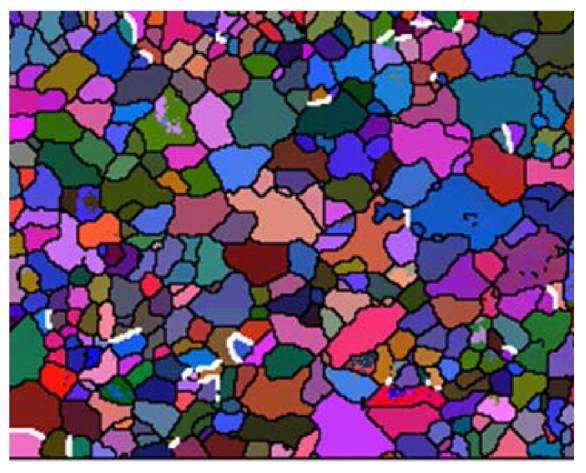

$0.5 \mathrm{~mm}$

(d) Au-Cu-Ag $+200 \mathrm{ppm} \mathrm{Ir}$

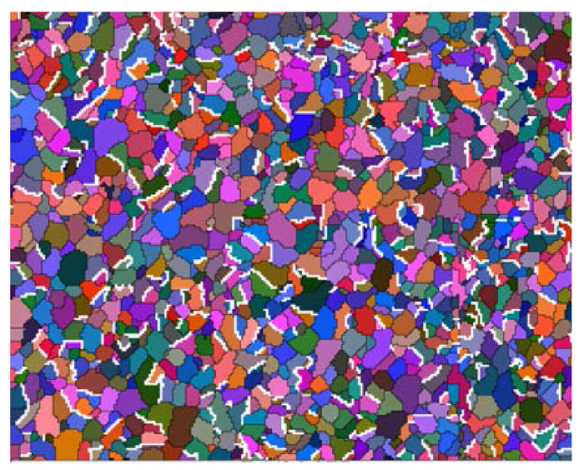

$\overline{0.25 \mathrm{~mm}}$

Fig. 2. EBSD false color reconstructed grain structures of samples solidified under nearly isothermal conditions showing the grain refinement induced by small additions of solute elements: (a) Al-20 wt.\%Zn; (b) Al-20 wt.\%Zn + 1000 ppm Cr; (c) Au-12.5 wt.\%Ag-12.5 wt.\%Cu; (d) Au12.5 wt. $\% A g-12.5$ wt. $\% \mathrm{Cu}+200$ ppm Ir. Further experimental details can be found in Refs. 1 and 2 .

the corresponding $\langle 110\rangle$ and $\langle 111\rangle$ pole figures in Fig. $3 b$ and c, respectively. The $\langle 110\rangle$ and $\langle 111\rangle$ directions of these grains are shown with the same color as those of the false-color map. The common $\langle 110\rangle$ direction of these five grains is circled in Fig. $3 b$, as well as for the five $\langle 111\rangle$ directions common to two nn grains in Fig. 3c. This fivefold MT symmetry, typical of decahedral symmetry (the decahedron is a base-element of an icosahedron), was explained in Refs. 1 and 2 by the heteroepitaxy relationship of the fcc phase on the triangular facets of icosahedral quasicrystals, as already mentioned in the previous section.

Figure 3d shows 4 grains in $\mathrm{Au}-\mathrm{Ag}-\mathrm{Cu}: \mathrm{Ir}$ with their corresponding $\langle 110\rangle$ and $\langle 211\rangle$ pole figures in (e) and (f), respectively. Again using the same color code for both the EBSD reconstructed grain structure and the pole figures, the pairs of grains A1/A2 and B1/B2 can be seen to be in a twin relationship (their common $\{111\}$ planes in (e) are shown with arcs of circle). Additionally, the pairs of grains $\mathrm{A} 1 / \mathrm{B} 1$ and A2/B2 share a common $\langle 110\rangle$ direction (circled in Fig. 3e), and the four grains share a common $\langle 211\rangle$ direction (double circled in Fig. 3f).
This situation is typical of four $\mathrm{nn}$ fcc grains having the heteroepitaxy relationship described before, with four adjacent facets of a so-called interlocked icosahedron, i.e., two icosahedra sharing a common pentagon. ${ }^{1,2}$

It could be argued that these observations can be explained by assuming direct nucleation of MT fcc nanoparticles from the melt, as observed in the 1960 s by $\operatorname{Ino}^{22,23}$ for pure gold nanoparticles formed in vacuum. Ino made an energy balance accounting for the anisotropic surface energy of $\mathrm{Au}$ and the strain energy associated with the assemblage of $20 \mathrm{fcc}$ tetrahedra in a MT icosahedral configuration. Indeed, an icosahedron/decahedron made of 20/5 fcc tetrahedra exhibits 20/10 external $\{111\}$ planes of minimum energy, but also elastic strain energy associated with missing gaps (the angle between the $\{111\}$ planes of a fcc tetrahedron being $70.53^{\circ}, 5$ undeformed tetrahedra in a decahedron configuration exhibit a missing gap of $7.35^{\circ}$ ). Ino calculated the density of strain energy, $g^{e \ell}$, associated with the deformation of $20 \mathrm{fcc}$ tetrahedra forming a MT icosahedron and obtained the following result: ${ }^{23}$ 
(a)

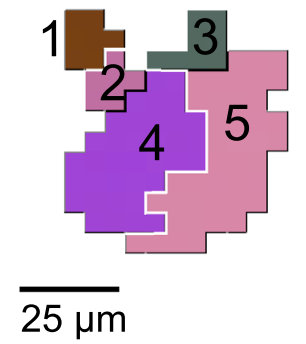

(d)

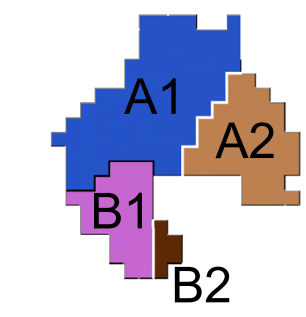

$100 \mu \mathrm{m}$
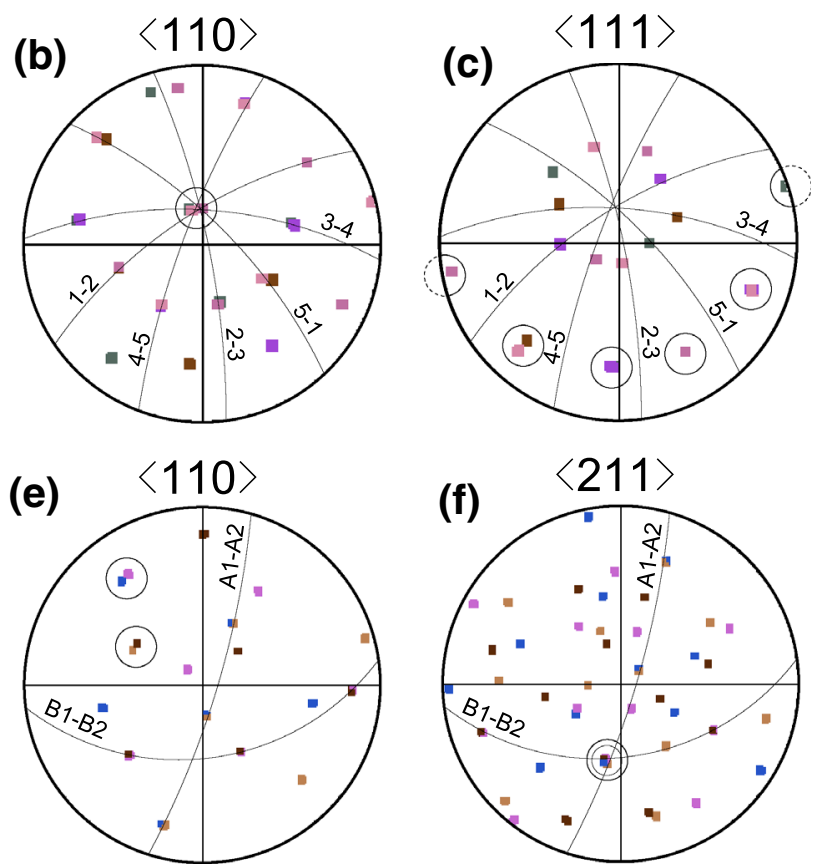

Fig. 3. (a) EBSD false color reconstruction of five nn fcc grains in a MT relationship in Al-Zn:Cr with their corresponding $\langle 110\rangle$ (b) and $\langle 111\rangle$ (c) pole figures. The traces of the twin (or near-twin) planes are drawn with arc of circles, while their corresponding $\langle 111\rangle$ directions and the $\langle 110\rangle$ direction common to all five grains are circled in (c) and (b), respectively. (d) EBSD false color reconstruction of four nn fcc grains in Au-Ag-Cu:Ir with their corresponding $\langle 110\rangle$ (e) and $\langle 211\rangle$ (f) pole figures. These grains exhibit the same symmetry as the four adjacent facets symmetrically positioned along the common pentagon of an interlocked icosahedron. The common $\{111\}$ twin plane to grains A1/A2 and B1/B2 are drawn with an arc of circle, the common $\langle 110\rangle$ directions of $\mathrm{A} 1 / \mathrm{B} 1$ and $\mathrm{A} 2 / \mathrm{B} 2$ are circled and the $\langle 211\rangle$ direction common to the four grains is double-circled in $(\mathbf{f})^{1,2}$.

$$
\begin{aligned}
g^{e \ell}= & \frac{1}{6}\left(\left(C_{11}+2 C_{12}\right)(2+v)^{2}+4 C_{44}(1-v)^{2}\right) \varepsilon_{x x}^{2} \\
& -3 \gamma_{G} k_{B} T(2+v) \varepsilon_{x x}
\end{aligned}
$$

with

$$
\begin{aligned}
v & =-\left(2 C_{11}+4 C_{12}-C_{44}\right) /\left(C_{11}+2 C_{12}+C_{44}\right) \\
\varepsilon_{x x} & =\frac{\delta}{1-(1+\delta) v} \quad \text { and } \quad \delta=0.080362
\end{aligned}
$$

where the $C_{i j}$ s are the fcc elastic constants, $\gamma_{G}$ is the Grüneisen coefficent and $\varepsilon_{x x}$ the deformation of each tetrahedron along a $\langle 110\rangle$ direction parallel to the $\{111\}$ outer surface necessary to close the gaps. Ino found that, below a critical radius, nanoparticles in vacuum have an energy advantage by exhibiting $\{111\}$ facets, despite this elastic strain energy contribution, whereas, above this value, Wulff equilibrium shapes are more favorable.

However, there are several reasons to argue against a direct transformation of liquid alloys into MT fcc nanoparticles. First, the same mechanism should occur without adding trace elements to the melt, a feature that is not observed. Second, because the solid-liquid interfacial energy is about onetenth of the solid surface energy, the advantage of minimizing the surface energy contribution by having $\{111\}$ planes in contact with the liquid would considerably reduce the radius below which icosahedral MT fcc nanoparticles are favored over regular Wulff crystals. For $\mathrm{Al}$ in vacuum, Ino estimated this transition radius to be about $3 \mathrm{~nm},{ }^{23}$ and so for a fcc MT particle in the liquid, this transition radius would be on the order of the atomic spacing, which does not make sense. Thirdly, the formation of quasicrystals in a melt is greatly facilitated compared to the direct nucleation of the fcc phase, since their interfacial energy with the liquid is about one order of magnitude lower ${ }^{24}$ (the homogeneous nucleation barrier depends on the cubic power of the interfacial energy). Finally, the fairly frequent occurrence of several nn grains in a twin configuration corresponding to that of an interlocked icosahedron (Fig. 3d-f) is fairly incompatible with a direct nucleation mechanism: liquid $\longrightarrow$ MT nanoparticles.

\section{FORMATION OF ICOSAHEDRAL QUASICRYSTALS IN THE MELT}

Frank suggested in the $1950 \mathrm{~s}^{3}$ that icosahedral short range order (ISRO) of atoms in the liquid could explain the large undercoolings measured by Turnbull $^{25}$ for various metals. However, the same ISRO can also explain the formation of $\mathrm{QCs}$ later observed by Shechtman et al. ${ }^{10}$ In Ref. 26 the following statement is made: "...under certain (composition and temperature) conditions, liquid metal 

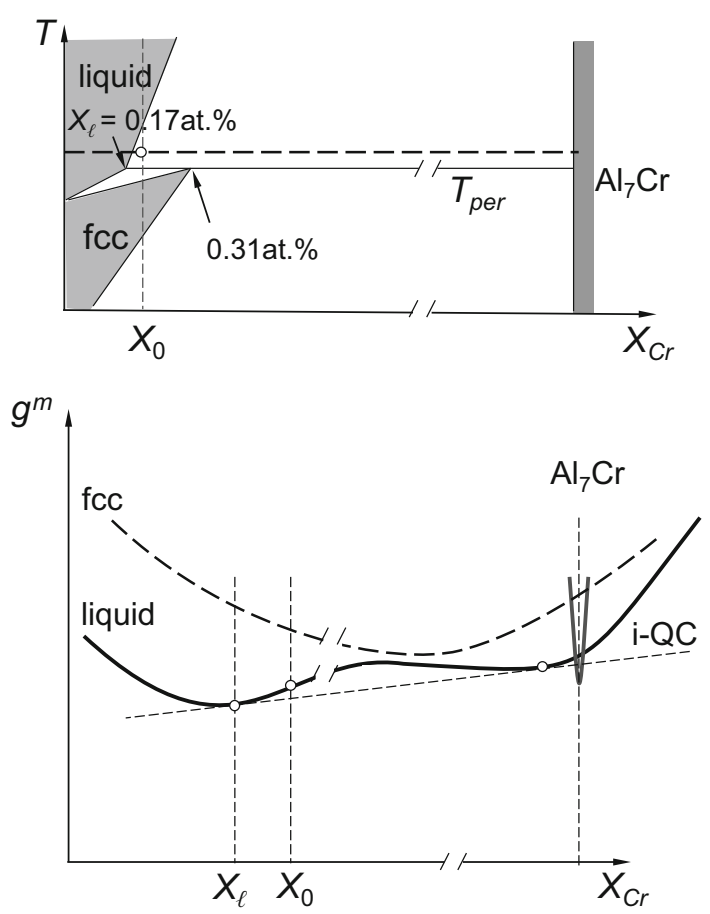

Fig. 4. Schematic partial view of the Al-Cr phase diagram (the temperature scale has been expanded) showing the single phase regions (top) and schematic view of the Gibbs free energies of fcc, liquid and $\mathrm{Al}_{7} \mathrm{Cr}$ at a temperature higher than the peritectic temperature $T_{\text {per }}$ (bottom). The nominal composition of the alloy, $X_{0}$, being very close to the equilibrium liquid composition, the curves have been interrupted just after $X_{0}$ and the maximum of the small hump (amplified in height) in fact should be much farther away from $X_{0}$.

alloys should contain a set of structural units (associates) that enter the nascent quasicrystal upon undercooling with minimal, if any, rearrangement; for a crystal to nucleate and grow, the associates should appreciably change their configuration or even be fully destroyed."

In the case of the Au-Ag-Cu:Ir system, ${ }^{2}$ the existence of stable QCs has not so far been reported, but the atomic radii of those elements, in particular that of Ir, is a good indicator that ISRO in the liquid can exist when iridium is added. Whether these icosahedral units ("associates" or building blocks) can or cannot cluster into small quasicrystals at fairly high temperature (but then disappear as the temperature decreases) remains an open question. Adding to the difficulty is that the phase diagram of yellow gold-iridium is very poorly known: $\mathrm{Cu}$-Ir is peritectic with a liquid composition at the peritectic temperature of about 11 wt.\%Ir. ${ }^{27}$ while Au-Ir is suspected to be eutectic but with a very small eutectic composition. ${ }^{28}$ Therefore, we will focus in this section on the Al-Zn:Cr system, since the phase diagrams (binaries and ternary) are fairly well known, and for the sake of simplicity we further restrict our discussion to the binary $\mathrm{Al}-\mathrm{Cr}$ system.

In this binary system, an approximant $\mathrm{Al}_{13} \mathrm{Cr}_{2}$ (also called $\mathrm{Al}_{7} \mathrm{Cr}$ or $\mathrm{Al}_{45} \mathrm{Cr}_{7}$ ) intermetallic phase, which exhibits many icosahedral building blocks in its large monoclinic cell, has been reported. ${ }^{14}$ Although there is some controversy on the peritectic versus eutectic nature of the phase diagram, we have shown clearly, using directional solidification experiments, that it is peritectic. ${ }^{29}$ In the ternary Al-20 wt.\%Zn:0.1 wt.\% Cr, the first liquidus to be encountered upon cooling is that of $\mathrm{Al}_{7} \mathrm{Cr},{ }^{1}$ reason why iQCs can form first in the melt. In the model binary Al-Cr system used for the sake of clarity, the composition of the liquid at the peritectic temperature is higher $\left(0.17\right.$ at. $\%$ or $\left.0.32 \mathrm{wt} . \%^{30}\right)$, and thus a $\mathrm{Cr}$ composition higher than this value is taken in order to have a similar situation. Since the temperature difference between the peritectic temperature and the melting point of pure $\mathrm{Al}$ is only about $1^{\circ} \mathrm{C}$, the phase diagram is only shown schematically in Fig. 4(top). Please note that the peritectic composition $\left(0.31\right.$ at. $\left.\%^{30}\right)$ is much smaller than that of the intermetallic $\mathrm{Al}_{7} \mathrm{Cr}$. The molar Gibbs free energy curves associated with this phase diagram are also shown schematically at the bottom of Fig. 4, for the purpose of discussion (the actual curves for the fcc and liquid phases are indeed very close to each other near the peritectic temperature in the range of composition of interest here, as will be shown in the next section).

Slightly above the peritectic temperature $T_{\text {per }}$ but below the liquidus of $\mathrm{Al}_{7} \mathrm{Cr}$, there is thermodynamic equilibrium between the liquid and $\mathrm{Al}_{7} \mathrm{Cr}$, the fcc phase Gibbs free energy curve, $G_{\mathrm{fcc}}^{m}$, being above the common tangent between $G_{\ell}^{m}$ and $G_{\mathrm{Al}_{7} \mathrm{Cr}}^{m}$. Even though the Gibbs free energy curve of the liquid $G_{\ell}^{m}$ is convex in the CALPHAD-type calculation of Ref. 30, it has been drawn with a small hump in Fig. 4. This is equivalent to stating that the icosahedral units with a $\mathrm{Cr}$ atom at the center present in the liquid tend to cluster into $\mathrm{Al}_{7} \mathrm{Cr}$ quasicrystals, in a manner similar to spinodal decomposition in a solid phase. With analogy to the formation of Guinier-Preston zones in solid state transformations, where for example $\mathrm{Cu}$-rich regions form in the fcc lattice of $\mathrm{Al}-\mathrm{Cu}$ alloys, this clustering of $\mathrm{Cr}$-centered icosahedra occurs with a very small interfacial energy, ${ }^{24}$ i.e., with a very small hump in the Gibbs free energy curve of $G_{\ell}^{m}$. The second (very weak) minimum of $G_{\ell}^{m}$ must occur at a composition close to the approximant phase $\mathrm{Al}_{7} \mathrm{Cr}$, and with an energy probably also very close, since for small clusters there is no need to distinguish quasicrystals from the approximant phase.

It should be pointed out that the $\mathrm{Cr}$ composition $X_{0}$ inducing grain refinement being close to the liquid composition at the peritectic temperature, $X_{\ell}\left(T_{\text {per }}\right)$, the formation of iQC by such a spinodal mechanism probably involves a nucleation growth process, rather than composition fluctuations of $\mathrm{Cr}$ icosahedral blocks. Assuming now a standard homogeneous nucleation law for a phase $v=$ fcc or $\mathrm{Al}_{7} \mathrm{Cr}$ iQC: 
Table I. Nucleation parameters for $\mathrm{Al}-\mathrm{Cr}$

\begin{tabular}{lcc}
\hline & Quasicrystal $\mathbf{A l} \mathbf{C r}_{\mathbf{7}} \mathbf{3 1 , 3 2}$ & fcc phase \\
\cline { 2 - 2 } Molar enthalpy of fusion $(\mathrm{J} / \mathrm{mole})$ & $13,390.0$ & 10,470 \\
Molar mass $(\mathrm{kg} / \mathrm{mole})$ & 0.03 & 0.027 \\
Entropy of fusion, $\Delta s_{f}^{v}(\mathrm{~J} / \mathrm{kg} / \mathrm{K})$ & 444.4 & 415.6 \\
Density, $\rho^{v}\left(\mathrm{~kg} / \mathrm{m}^{3}\right)$ & 2983.0 & 2550.0 \\
Interfacial energy with liquid, $\gamma_{v \ell}\left(\mathrm{J} / \mathrm{m}^{2}\right)$ & $0.016^{a}$ & 0.16 \\
Pre-exponential factor, $I_{0}^{v}\left(\mathrm{~J} / \mathrm{m}^{3} / \mathrm{s}^{1}\right)$ & $4.8 \times 10^{38, b}$ & $1.0 \times 10^{42}$ \\
\hline
\end{tabular}

${ }^{\mathrm{a}} \gamma_{\mathrm{QC} / \ell}$ has been taken one order of magnitude lower than $\gamma_{\mathrm{fcc} / \ell}$ based on Ref. $24 .^{\mathrm{b}} I_{0}^{\mathrm{QC}}$ is based on the density of Cr atoms in the liquid.

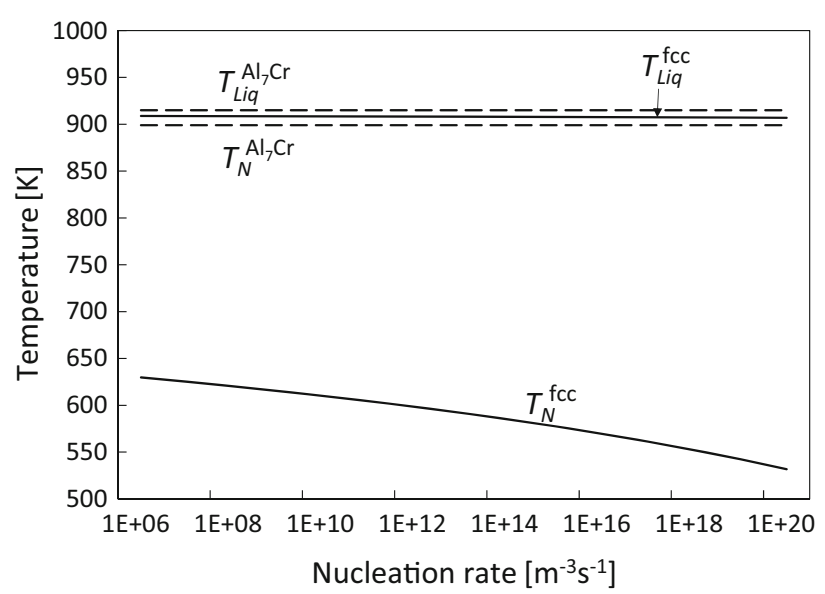

Fig. 5. Calculated liquidus temperature, $T_{\text {Liq }}$, and temperature of nucleation, $T_{N}(I)$, of the fcc (continuous lines) and $\mathrm{Al}_{7} \mathrm{Cr}$ iQC phases (dashed lines) in Al-20 wt.\%Zn:0.1 wt.\%Cr, as a function of the nucleation rate $l$.

$$
I^{v}(T)=I_{0}^{v} \exp \left(-\frac{16 \pi}{3} \frac{\gamma_{v \ell}^{3}}{\left(\rho_{v} \Delta s_{f}^{v} \Delta T\right)^{2} k_{B} T}\right)
$$

we can compute the temperature $T_{N}\left(I^{v}\right)$ at which a certain nucleation rate $I^{v}$ occurs for phase $v$. The data used for this calculation are listed in Table I. The pre-exponential factor $I_{0}^{v}$ is based on the density of atoms in the liquid for the fcc phase, whereas it assumes that only $\mathrm{Cr}$ atoms can initiate a cluster of the iQC phase. The volumetric entropy of fusion for the transformation, $\rho_{v} \Delta s_{f}^{v}$, of both phases is not drastically different, and the undercooling $\Delta T$ for each phase is given by $\left(T_{\mathrm{Liq}}^{v}-T\right)$. While the preexponential factors differ by three orders of magnitude, the most important difference between the two phases is in fact the interfacial energy, $\gamma_{v \ell}: \gamma_{\mathrm{QC} / \ell}$ has been taken one order of magnitude lower than $\gamma_{\mathrm{fcc} / \ell}{ }^{24}$ The result of this calculation, which is only approximate, is shown in Fig. 5. As can be seen, while typically $300 \mathrm{~K}$ is required to have a substantial amount of homogeneous fcc nuclei formed in the melt, only $7 \mathrm{~K}$ is necessary for the $\mathrm{iQC}$ phase.
This result simply reflects the factor of 10 difference in the interfacial energy, i.e., a factor of 1000 in the exponential argument, despite the fact that the density of atoms which has to be considered for iQC cluster formation (i.e., chromium for $\mathrm{Al}-\mathrm{Zn}$ ) is more than $10^{3}$ lower. The temperature at which the iQCs nucleate is just below the liquidus of the fcc phase and thus if the $\mathrm{iQC}$ can grow to a sufficient size, they can promote the formation of the fcc phase by an heteroepitaxy mechanism.

\section{iQC-ENHANCED NUCLEATION MECHANISM: HETEROEPITAXY SOLIDIFICATION}

The heteroepitaxy mechanism suggested in Refs. 1 and 2 is summarized in Fig. 1. After nucleation at about 7-10 $\mathrm{K}$ undercooling for $\mathrm{Al}-\mathrm{Zn}$ :Cr (i.e., a critical radius on the order of 1-2 $\mathrm{nm}$ ), the iQCs grow by aggregation of icosahedral building blocks in the liquid. For that purpose, chromium has to diffuse toward the growing iQC phase. Due to the peritectic nature of the phase diagram, ${ }^{29}$ the liquid surrounding the iQCs is depleted in chromium. According to the suggested mechanism, once the $\mathrm{iQC}$ reaches a radius $R_{\mathrm{QC}}$ and an undercooling $\Delta T$ below the liquidus of the fcc phase, this phase can form on the triangular facets of the $\mathrm{iQC}$, with twins (or near-twins) in between the 20 fcc nuclei. Assuming a nearly-spherical fcc MT particle of radius $R$, with an internal iQC particle in its center, the energy balance for the formation of the fcc phase is given by:

$$
\begin{aligned}
\Delta G_{\mathrm{fcc}}^{\mathrm{QChetero}}= & \left(-\rho^{\mathrm{fcc}} \Delta s_{f}^{\mathrm{fcc}} \Delta T+g^{e \ell}\right) \frac{4 \pi}{3}\left(R^{3}-R_{\mathrm{QC}}^{3}\right) \\
& +4 \pi R^{2} \gamma_{\mathrm{fcc} / \ell}+4 \pi R_{\mathrm{QC}}^{2}\left(\gamma_{\mathrm{QC} / \mathrm{fcc}}-\gamma_{\mathrm{QC} / \ell}\right)
\end{aligned}
$$

where $\gamma_{\mathrm{QC} / \mathrm{fcc}}$ is the interfacial energy between the $\mathrm{QC}$ and the fcc phase, $\Delta T$ is the undercooling of the fcc phase and $g^{e \ell}$ is the volumetric strain energy associated with the formation of 20 MT fcc tetrahedra as described previously. Differentiating Eq. 3 with respect to $R$ to find the maximum of the energy barrier, we retrieve naturally the value of the 
critical radius of a spherical fcc nucleus in equilibrium with the liquid (i.e., the Gibbs-Thomson relationship), but in which the driving force $\left(\rho^{\mathrm{fcc}} \Delta s_{f}^{\mathrm{fcc}} \Delta T\right)$ is decreased by $g^{e \ell}$ :

$$
\begin{aligned}
R^{*}= & \frac{2 \Gamma_{\mathrm{fcc} / \ell}}{\Delta T-g^{e \ell} /\left(\rho^{\mathrm{fcc}} \Delta s_{f}^{\mathrm{fcc}}\right)} \\
& \text { with } \quad \Gamma_{\mathrm{fcc} / \ell}=\frac{\gamma_{\mathrm{fcc} / \ell}}{\rho^{\mathrm{fcc}} \Delta s_{f}^{\mathrm{fcc}}}
\end{aligned}
$$

The strain energy contribution, which plays here a role similar to that involved in the nucleation of coherent precipitates in a supersaturated solid solution, can be estimated from the work of Ino. ${ }^{22,23}$ This author estimated that, at room temperature, the contribution $g^{e l}$ for a MT icosahedron of Al is about $9.8 \times 10^{7} \mathrm{~J} / \mathrm{m}^{3}$. Taking the elastic modulus near the melting point to be about half the value at room temperature, we can reasonably assume that the contribution $g^{\text {el }}$ in Eq. 4 is on the order of $5 \times 10^{7} \mathrm{~J} / \mathrm{m}^{3}$. Since the volumetric entropy of fusion of the fcc Al phase, $\left(\rho \Delta s_{f}\right)^{\mathrm{fcc}}$, is only $10^{6} \mathrm{~J} / \mathrm{m}^{3} / \mathrm{K}$, we may conclude that a minimum of $50 \mathrm{~K}$ undercooling would be required to overcome the elastic energy, regardless of the surface energy contribution. Since the iQC-enhanced nucleation in $\mathrm{Al}-\mathrm{Zn}$ : $\mathrm{Cr}$ has been observed at about $10 \mathrm{~K}$ undercooling, ${ }^{1}$ we conclude that direct transformation of the liquid into $20 \mathrm{MT}$ nuclei on the facets of the iQC is unlikely, unless the elastic energy contribution is minimized by an appropriate choice of atom sizes in the alloy which would compensate for the missing gaps.

In addition to the elastic energy contribution, let us now focus on the surface energy contributions (assuming $g^{e \ell} \cong 0$ ). Since $\gamma_{\mathrm{QC} / \ell}$ is very small (typically $1 / 10$ to $1 / 50$ of $\gamma_{\mathrm{fcc} / \ell},{ }^{24}$ ) building an fcc layer around the initial QC-liquid interface can only increase the surface energy contribution since we add the contributions $\gamma_{\mathrm{fcc} / \ell}$ and $\gamma_{\mathrm{QC} / \mathrm{fcc}}$. With the assumed heteroepitaxy relationship, this last contribution must be relatively small, probably on the order of $\gamma_{\mathrm{QC} / \ell}$. Neglecting the last term in Eq. 3 associated with the QC-fcc interface and using the result found in Eq. 4, the energy barrier associated with iQC-enhanced nucleation of an fcc layer is then given by:

$$
\Delta G_{\mathrm{fcc}}^{\mathrm{QChetero}}=\Delta G_{\mathrm{fcc}}^{\mathrm{homo}}\left[1+\frac{3}{4}\left(\frac{\Delta T R_{\mathrm{QC}}}{\Gamma_{\mathrm{fcc} / \ell}}\right)^{3}\right]
$$

where $\Delta G_{\mathrm{fcc}}^{\mathrm{homo}}=16 \pi \gamma_{\mathrm{fcc}, \ell}^{3} /\left[3\left(\rho^{\mathrm{fcc}} \Delta s_{f}^{\mathrm{fcc}} \Delta T\right)^{2}\right]$ is the homogeneous nucleation barrier for the same driving force of a full fcc sphere of radius $R^{*}$. As can be seen, the energy barrier in this case is larger, and not lower, than that of homogeneous nucleation. This situation contrasts with that of heterogeneous nucleation of a spherical fcc cap on a flat surface. ${ }^{25}$ This can easily be understood since the formation of a spherical fcc shell around a spherical foreign substrate (here the iQC) is favored only if the interfacial energy between this substrate (QC) and the liquid is large compared to the two other interfacial energies appearing in Eq. 3, in other words if $\gamma_{\mathrm{QC} / \ell}>\left(\gamma_{\mathrm{fcc} / \ell}+\gamma_{\mathrm{QC} / \mathrm{fcc}}\right)$, which is the opposite of what has been assumed. A similar calculation with triangular flat fcc layers forming on the iQC (instead of an overall spherical fcc phase with an nearly spherical $\mathrm{iQC}$ at the center) leads to the same conclusion: the fcc minimum layer thickness $\delta$ giving a positive energy balance at around $\Delta T \cong 10 \mathrm{~K}$ (without the elastic energy contribution) is given by:

$$
\delta>\frac{\gamma_{\mathrm{fcc} / \ell}+\gamma_{\mathrm{QC} / \mathrm{fcc}}-\gamma_{\mathrm{QC} / \ell}}{\rho^{\mathrm{fcc}} \Delta s_{f}^{\mathrm{fcc}} \Delta T} \cong 10 \mathrm{~nm}
$$

which is too big for any reasonable nucleation mechanism.

In summary, a transformation of a liquid layer surrounding the $\mathrm{iQC}$ formed at low undercooling into an fcc shell would require an undercooling much larger than the $10 \mathrm{~K}$ measured in DSC for $\mathrm{Al}-\mathrm{Zn}: \mathrm{Cr}^{1}$ for two reasons: (i) the elastic energy contribution already requires about $50 \mathrm{~K}$ undercooling for a pure Al fcc phase forming around the iQC; (ii) the new interface between the fcc and the liquid has a much higher interfacial energy (about an order of magnitude) than that between the iQC and the liquid. Therefore, other considerations have to be examined.

\section{iQC-ENHANCED NUCLEATION MECHANISM: PERITECTIC TRANSFORMATION}

Since the volumetric driving force at $\Delta T=10 \mathrm{~K}$ undercooling, given by $\left(\rho \Delta s_{f}\right)^{\mathrm{fcc}} \Delta T$, appears insufficient for transforming the liquid around the iQC into an MT fcc layer, we now consider the peritectic transformation of a layer of $\mathrm{iQC}$ into fcc. Indeed, just above the peritectic temperature, the $\mathrm{iQC}$ is in equilibrium

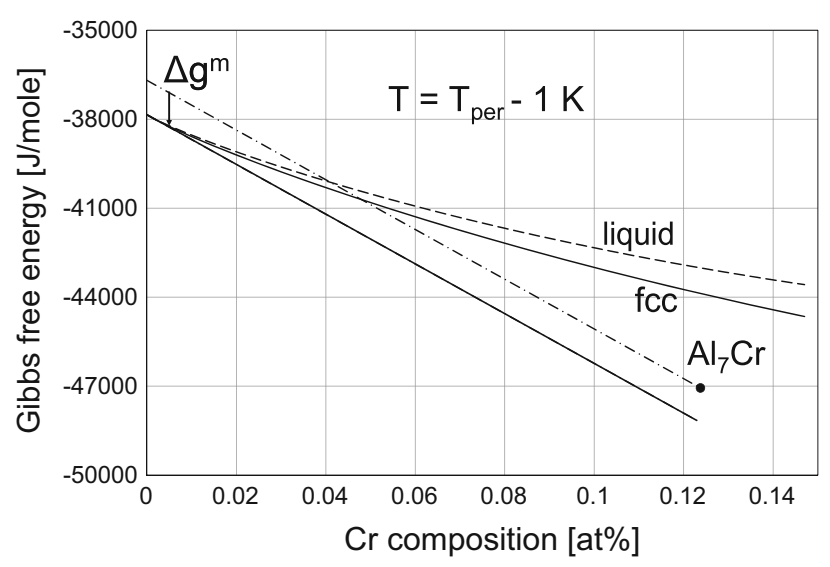

Fig. 6. Molar Gibbs free energy curves $1 \mathrm{~K}$ below the peritectic temperature of $\mathrm{Al}-\mathrm{Cr}$ for the liquid (dashed line), fcc phase (solid line) and $\mathrm{Al}_{7} \mathrm{Cr}$ (black point). This last phase has been taken as stoichiometric and the common tangent to the solid and liquid energy curves has been drawn. The thermodynamic data are taken from Ref. 30. 
with the liquid, while just below it must be replaced by the fcc phase. The question remains on how the peritectic phase (the fcc phase in this case) nucleates.

Using the data of Ref. 30 for the Al-Cr binary alloy, Fig. 6 shows the Gibbs free energies of the fcc phase (solid curve), of the liquid (dashed curve) and of the approximant $\mathrm{Al}_{7} \mathrm{Cr}$ intermetallic phase (black dot), for a temperature only $1 \mathrm{~K}$ below the peritectic invariant. At this temperature, the fcc and liquid phases are in equilibrium for a composition $X_{\mathrm{Cr}}=0$, i.e., the temperature is equal to the melting point of pure aluminum. Please note that the $\mathrm{Al}_{7} \mathrm{Cr}$ phase has been assumed to be stoichiometric. The common tangent to the fcc and liquid Gibbs free energies is also drawn: it passes through the Gibbs free energy of pure $\mathrm{Al}$. As can be seen, the energy of the intermetallic is more than $1000 \mathrm{~J} /$ mole above this common tangent, i.e., a factor 100 compared to the driving force for a liquid-to-fcc transformation at an undercooling of $1 \mathrm{~K}\left(\Delta s_{f}^{\mathrm{fcc}, m} \Delta T=10 \mathrm{~J} / \mathrm{mole}\right)$.

Baker and $\mathrm{Cahn}^{33}$ gave the general thermodynamic rule governing the transfer of a small volume element of composition $X_{r}$ from a parent phase $\beta$ of composition $X_{\beta}$ to a product phase $\alpha$ of composition $X_{\alpha}$ : the driving force is given by the Gibbs free energy difference at the composition $X_{r}$ between the two tangents drawn for $\beta$ at $X_{\beta}$ and $\alpha$ at $X_{\alpha}$. In the case of an intermetallic phase of stoichiometric composition, this rule cannot be applied. If the phase has a small solubility range, the Gibbs free energy must quickly rise once the composition goes off this range. In the $\mathrm{Al}-\mathrm{Cr}$ system, the chromium composition of the intermetallic must slightly decrease during growth since the liquid becomes leaner in chromium: this slope can thus quickly tend to $-\infty$. Taking not such an extreme situation, we have chosen a moderate slope equal to that of the fcc-liquid common tangent in Fig. 6. Under such condition, an outer layer of an iQC containing no (or little) chromium can transform into fcc with the driving force $\Delta g^{m}$ shown in this figure. This very large driving force can overcome without any difficulty the strain energy associated with multiple twins and the increase of the interfacial energy. Of course, this fcc layer should now be stable with respect to the melt, i.e., the construction in Fig. 6 should be made with a Gibbs free energy curve of the fcc phase shifted up to account for curvature. Measuring by DSC the solidification peak of the fcc phase at an undercooling of $10 \mathrm{~K}$ means that this radius is on the order of $20 \mathrm{~nm}$. Finally, the construction shown in Fig. 6 does not tell how diffusion of chromium proceeds further, but it is clear that the peritectic fcc phase must dissolve the intermetallic (peritectic transformation) while solidifying at the same time.

\section{CONCLUSION}

As a first conclusion, a thermodynamic approach to the iQC-enhanced nucleation of the fcc phase in liquid metals seems to indicate that:
- Nucleation of iQC in the liquid can occur at low undercooling due to the reduced interfacial energy. An order of magnitude difference in $\gamma_{\mathrm{QC} / \ell}$ compared to $\gamma_{\mathrm{fcc} / \ell}$ induces a drastic change of the undercooling at which an appreciable density of nuclei form, despite the reduced density of atoms around which nucleation can occur. The critical radius at which these $\mathrm{iQCs}$ form is estimated to be on the order of $\mathrm{nm}$.

- The iQCs then grow, but because the phase diagram is peritectic, the melt becomes depleted in solute atoms that are at the center of the icosahedral building blocks.

- Below the peritectic temperature, the iQCs no longer correspond to the stable phase and should disappear. We have used Baker and Cahn's construction to evaluate the driving force for the transformation of an outer layer of the iQCs very lean in chromium atoms into fcc. This driving force can be two orders of magnitude larger than that associated with the solidification of this layer, thus giving the possibility to overcome the strain energy associated with multiple twins and the interfacial energy increase at a moderate undercooling. In order to produce a stable fcc layer with respect to the liquid, the radius of the fcc layer at the periphery of the iQCs must be large enough. Taking an order of magnitude for the interfacial energy difference between $\mathrm{iQC}$ and fcc, this means that the $\mathrm{iQC}$ must grow by at least a factor of 10 prior to this peritectic transformation.

- After nucleation of the peritectic fcc layer, the iQC template can be consumed by the peritectic transformation while the liquid further solidifies into fcc, as expected for a peritectic alloy.

This conclusion should be moderated by the following arguments. Thermodynamics is a science based on a sufficiently large population of atoms over which average quantities, such as molar Gibbs free energy or surface energy, can be defined. In nucleation theories, we know that such is not the case, the critical nucleus being typically on the order of a nm or even less, i.e., a value on the order of the diffuse solid-liquid interface thickness. What is then the meaning of distinguishing an excess Gibbs free energy for atoms located at "the" surface of the nucleus, i.e., the surface energy concept, from the bulk Gibbs free energy? This certainly shows a general limit of the applicability of any thermodynamic approach to nucleation, especially when the material has a diffuse interface at the atomic scale. Therefore, we can see the limits of the present thermodynamic approach to the present nucleation mechanisms. In this respect, the deposition of metallic atoms onto QC clean surfaces under ultrahigh vacuum by Fournée et al.$^{34}$ clearly shows how complex the nucleation mechanisms can be. In order to further elucidate this iQC-mediated nucleation mechanism during solidification, ab initio/MD 
calculations and maybe in situ diffraction experiments should be performed.

Nevertheless, we believe that the consideration made in here provides some guidelines for this efficient nucleation mechanism to occur. First, the atomic sizes of the various solvent and solute elements must be such that icosahedral short range order in the liquid occurs. This can be verified typically by looking at the atomic structure of the approximant phase, or by atomistic calculations. Second, the nominal composition of the alloy must be such that the liquidus of the approximant intermetallic phase is first encountered. In fact it should be large enough so that nucleation of the iQCs can occur before typically reaching the peritectic temperature, typically about $10 \mathrm{~K}$ above the peritectic. Third, the composition should not be too high, otherwise the iQC will become too big and may even switch to the crystalline approximant phase. While there are many studies on the heteroepitaxy relationship between $\mathrm{iQC}$ and its approximant phase, it is not certain that the approximant can induce heteroepitaxy of the fcc phase. Furthermore, below the peritectic temperature, the intermetallic phase might be too large to allow the transformation into a MT fcc structure because the elastic energy involved will then become prohibitive. In summary, the alloy composition should be such that icosahedral short range order occurs and the liquidus of the approximant phase must be about $10 \mathrm{~K}$ above the peritectic temperature, but not much more. Such predictions should be validated further on industrial aluminum alloys which are prone to twinned dendrites formation. In such alloys, trace elements such as $\mathrm{Cr}$ and $\mathrm{Ti}$, which have a low peritectic composition, but also $\mathrm{Mn}$ or $\mathrm{Fe}$, which have a low eutectic composition and for which QCs have been reported, ${ }^{24}$ could trigger an iQC-mediated nucleation mechanism leading to their formation.

\section{ACKNOWLEDGEMENTS}

The authors thank Michele Ceriotti, Jon Dantzig and Wilfried Kurz for many helpful discussions, as well as Rolex and Constellium for their financial support.

\section{REFERENCES}

1. G. Kurtuldu, P. Jarry, M. Rappaz, Acta Mater. 61, 7098 (2013).
2. G. Kurtuldu, A. Sicco, M. Rappaz, Acta Mater. 70, 240 (2014).

3. F.C. Frank, Proc. R. Soc. Lond. A Math. Phys. Sci. 215, 43 (1952).

4. P.J. Steinhardt, D.R. Nelson, M. Ronchetti, Phys. Rev. B 28, 784 (1983).

5. X. Fang, C. Wang, Y. Yao, Z. Ding, K. Ho, Phys. Rev. B 83, 224203 (2011).

6. H. Reichert, O. Klein, H. Dosch, M. Denk, V. Honkimäki, T. Lippmann, G. Reiter, Nature 408, 839 (2000).

7. V. Simonet, F. Hippert, M. Audier, R. Bellissent, Phys. Rev. B 65, 024203 (2001).

8. T. Schenk, D. Holland-Moritz, V. Simonet, R. Bellissent, D. Herlach, Phys. Rev. Lett. 89, 075507 (2002).

9. D. Holland-Moritz, T. Schenk, R. Bellissent, V. Simonet, K. Funakoshi, J. Merino, T. Buslaps, S. Reutzel, J. Non Cryst. Solids 312, 47 (2002).

10. D. Shechtman, I. Blech, D. Gratias, J.W. Cahn, Phys. Rev. Lett. 53, 1951 (1984).

11. H. Zhang, D. Wang, K. Kuo, Phys. Rev. B 37, 6220 (1988).

12. H. Zhang, D. Wang, K. Kuo, J. Mater. Sci. 24, 2981 (1989).

13. A. Srivastava, S. Ranganathan, Acta Mater. 44, 2935 (1996).

14. M. Cooper, Acta Crystallogr. 13, 257 (1960).

15. Z. He, B. Zou, K. Kuo, J. Alloys Compd. 417, L4 (2006).

16. K. Kelton, G. Lee, A. Gangopadhyay, R. Hyers, T. Rathz, J. Rogers, M. Robinson, D. Robinson, Phys. Rev. Lett. 90, 195504 (2003).

17. S. Henry, M. Rappaz, P. Jarry, Metall. Mater. Trans. A 29, 2807 (1998).

18. M. Salgado-Ordorica, M. Rappaz, Acta Mater. 56, 5708 (2008).

19. J.P. Nielsen, J. Tuccillo, J. Dent. Res. 45, 964 (1966).

20. D. Ott, C.J. Raub, Gold Bull. 14, 69 (1981).

21. H. Renner, G. Schlamp, D. Hollmann, H. M. Lüschow, P. Tews, J. Rothaut, K. Dermann, A. Knödler, C. Hecht, M. Schlott, R. Drieselmann, C. Peter, and R. Schiele, Ullmann's Encyclopedia of Industrial Chemistry (Wiley-VCH, Weinheim, 2000).

22. S. Ino, J. Phys. Soc. Jpn. 21, 346 (1966).

23. S. Ino, J. Phys. Soc. Jpn. 27, 941 (1969).

24. K. Kelton, Int. Mater. Rev. 38, 105 (1993).

25. D. Turnbull, J. Appl. Phys. 21, 1022 (1950).

26. A. Zaitsev, N. Zaitseva, E.K. Shakhpazov, N. Arutyunyan, S. Dunaev, Doklady Physical Chemistry, vol. 406 (Springer, Berlin, 2006).

27. D. Chakrabarti, D. Laughlin, J. Phase Equilib. 8, 132 (1987).

28. M. Riabkina, L. Gal-Or, Y. Fishman, G. Iram, Gold Bull. 17, 62 (1984).

29. G. Kurtuldu, P. Jessner, M. Rappaz, J. Alloys Compd. 621, 283 (2015).

30. Y. Liang, C. Guo, C. Li, Z. Du, J. Alloys Compd. 460, 314 (2008).

31. O. Kubaschewski, G. Heymer, Trans. Faraday Soc. 56, 473 (1960).

32. V.S. Zolotorevsky, N.A. Belov, M.V. Glazoff, Casting Aluminum Alloys (Elsevier, Amsterdam, 2010).

33. J.C. Baker, J.W. Cahn, The Selected Works of John W. Cahn (2013).

34. V. Fournée, J. Ledieu, M. Shimoda, M. Krajčí, H.-R. Sharma, R. McGrath, Isr. J. Chem. 51, 1314 (2011). 